\title{
Use of an over-the-scope clip for endoscopic sealing of anastomotic dehiscence after anterior resection
} for rectal cancer

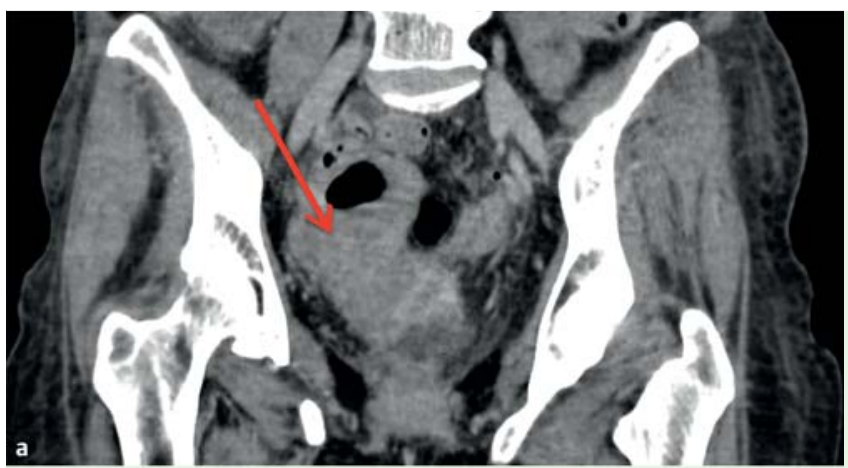

Fig. 1 Computed tomographic scans of a 4-cm para-anastomotic abscess (arrows) in a patient who 15 days earlier had undergone anterior resection of the rectum for distal rectal adenocarcinoma. a Coronal view. b Axial view.

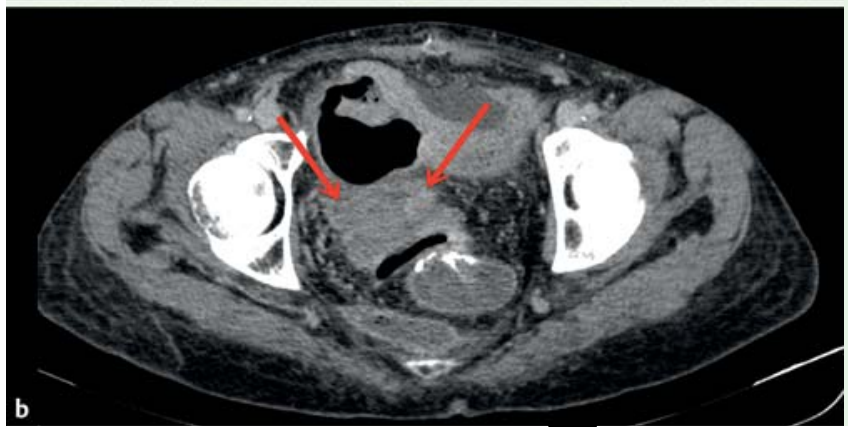

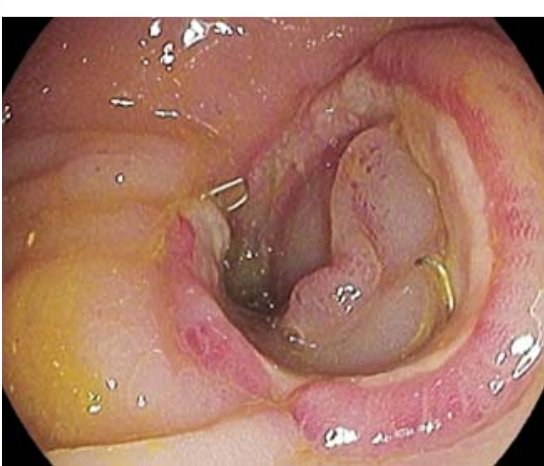

Fig. 2 Proctosigmoidoscopy confirms a 12-mm orifice along the right edge of the rectal anastomosis, $3 \mathrm{~cm}$ from the pectinate line.

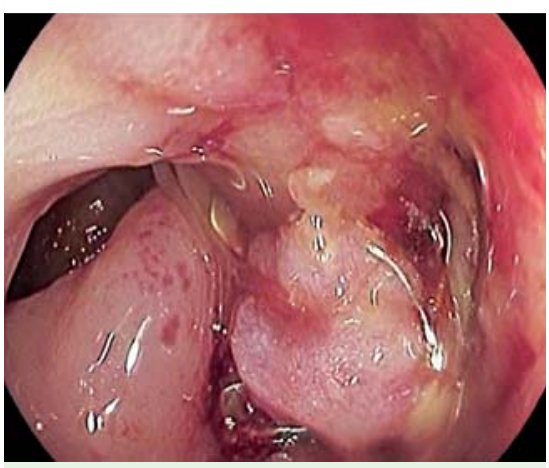

Fig. 4 First image of leak closure after overthe-scope clip deployment.
Oncological colo-rectal surgery may be complicated by anastomotic leakage, especially in patients who have undergone anterior resection for distal cancer. Occurring in up to $20 \%$ of such patients [1], anastomotic leakage usually requires emergency intervention with a prolonged hospital stay, adversely affects both longterm survival and quality of life, and may result in the need for a permanent stoma $[1,2]$.

Here, we report the case of a 72-year-old woman presenting with fever 15 days after undergoing a laparotomy with anterior resection of the rectum, total mesorectal excision, and Knight-Griffen reconstruction for distal rectal adenocarcinoma (pT4N1M0 G2). Computed tomography revealed a 4-cm para-anastomotic abscess ( $\bullet$ Fig. 1). Proctosigmoidoscopy confirmed a $12-\mathrm{mm}$ orifice along the right edge of the rectal anastomosis, $3 \mathrm{~cm}$ from the pectinate line ( $\mathbf{F i g . 2}$ ). Based on the size and position of the leak, it was decided to place an over-the-scope clip (OTSC; Ovesco Endoscopy, Tübingen, Germany) for dehiscence sealing.

First, the endoscope was withdrawn, and an OTSC with a 6-mm depth and pointed teeth was mounted on its tip [3]. Afterward, an "anchor" device (Ovesco) was advanced through the working channel of the scope toward the leak edges. Maneuvering the scope with gentle axial turning allowed correct placement of the anchor ( $\bullet$ Fig. 3 ); then, the anchor was pulled into the distal cap of the OTSC system under continuous suction.

The OTSC was deployed after more than half of the volume of the clear distal cap was filled with tissue, as previously described [4]. The OTSC setup up took about 3 minutes, after which endoscopic views revealed complete closure of the leak despite maximal insufflation ( $\mathrm{Fig} .4$ and - Fig.5). A few hours later, a laparotomy with peritoneal lavage was performed to drain the pelvic abscess, with two peritoneal drains left in place. The patient's clinical condition quickly improved, and spontaneous bowel movements resumed 72 hours later. Accordingly, the patient was discharged 7 days later.

This case is interesting for several reasons. First, we have for the first time demonstrated the use of an OTSC to manage a distal rectal dehiscence and have also provided a detailed description of the procedure, which may be helpful in future cases. Second, we have shown that the endoscopic sealing of a distal rectal anastomotic dehiscence is feasible, and combined with 
a conservative surgical approach, it can avoid the need for a redo anastomosis or a permanent stoma. Finally, our report adds to the growing literature on the potential applications of OTSCs $[4,5]$. Indeed, the recently introduced OTSC system provides a practical alternative for the treatment of low rectal anastomotic leakage, in which standard surgical approaches are associated with considerable risks and require aggressive strategies.

Endoscopy_UCTN_Code_CPL_1AJ_2AG

Competing interests: None

\section{Paola Soriani ${ }^{1,2}$, Gian Eugenio Tontini ${ }^{1}$, Luca Pastorelli, ${ }^{1,3}$, Massimo Mauri ${ }^{4}$, Helmut Neumann ${ }^{5}$, Maurizio Vecchi ${ }^{1,3}$, Pavlos Lagoussis ${ }^{4}$}

${ }^{1}$ Gastroenterology and Digestive Endoscopy Unit, IRCCS Policlinico San Donato, San Donato Milanese, Italy

${ }^{2}$ Gastroenterology Unit, Department of Medicine, University of Parma, Parma, Italy
${ }^{3}$ Department of Biomedical Sciences for Health, University of Milan, Milan, Italy

${ }^{4}$ Division of General Surgery I, IRCCS Policlinico San Donato, San Donato Milanese, Italy

${ }^{5}$ Department of Medicine I, University of Erlangen-Nuremberg, Erlangen, Germany

\section{References}

1 Wu SW, Ma CC, Yang Y. Role of protective stoma in low anterior resection for rectal cancer: a meta-analysis. World J Gastroenterol 2014; 20: 18031 - 18037

2 Pitel S, Lefèvre JH, Tiret $E$ et al. Redo coloanal anastomosis: a retrospective study of 66 patients. Ann Surg 2012; 256: 806-810

3 Banerjee S, Barth BA. ASGE Technology Committee et al. Endoscopic closure devices. Gastrointest Endosc 2012; 76: 244 - 251

4 Tontini GE, Naegel A, Albrecht $H$ et al. Successful over-the-scope clip (OTSC) treatment for severe bleeding due to anastomotic dehiscence. Endoscopy 2013; 45 (Suppl. 02): E343-E344

5 Mönkemüller K, Peter S, Toshniwal J et al. Multipurpose use of the "bear claw" (overthe-scope-clip system) to treat endoluminal gastrointestinal disorders. Dig Endosc 2014; 26: $350-357$
Bibliography

DOI http://dx.doi.org/

10.1055/s-0034-1391875

Endoscopy 2015; 47: E278-E279

(c) Georg Thieme Verlag KG

Stuttgart · New York

ISSN 0013-726X

\section{Corresponding author}

\section{Gian Eugenio Tontini, MD, PhD}

Gastroenterology and Digestive Endoscopy Unit IRCCS Policlinico San Donato

Via Morandi 30

20097, San Donato Milanese (MI)

Italy

Fax: +39-02-52774655

gianeugeniotontini@libero.it 